\title{
Slim exceptional sets for sums of fourth and fifth powers
}

by

\author{
Koichi Kawada (Morioka) and Trevor D. Wooley (Ann Arbor, MI)
}

1. Introduction. In previous memoirs [12], [13] devoted to the topic of effective exploitation of excess variables in exceptional set estimates relevant to Waring's problem, the second author reported on investigations involving sums of squares, and sums of cubes, respectively. The purpose of this paper is to describe how such methods may be applied for certain higher powers, illustrating our ideas en route with a selection of basic examples intended to provide models for future forays by enthusiasts. The ideas underlying our methods are clearly illustrated in [12] and [13], so for the moment we content ourselves by noting only that our methods avoid a conventional application of Bessel's inequality in favour of explicit control of an exponential sum over the exceptional set itself. Such ideas also play an important role in recent work of the authors joint with Brüdern (see [1], for example) devoted to the representation primarily of thin polynomial sequences as sums of powers of natural numbers. We remark that the ideas underlying our methods are simple and concise, qualities that would be easily masked by a detailed account of the technical aspects of our analysis. We have therefore opted for an abbreviated exposition that makes free use of results familiar to aficionados of the modern theory of Waring's problem.

We begin in $\S \S 2-5$ with an investigation of exceptional sets associated with sums of biquadrates, and this entails a discussion of the associated local solubility conditions. For every integer $x$ one has $x^{4} \equiv 0$ or 1 modulo 16 , and so it follows that whenever $n$ is the sum of $s$ biquadrates, then necessarily $n \equiv r(\bmod 16)$ with $r$ an integer satisfying the condition $0 \leq r \leq s$. Moreover, if $n$ is the sum of $s<16$ biquadrates and $16 \mid n$, then $n / 16$ is also the sum of $s$ biquadrates. In investigations concerning the representation of integers $n$ as sums of $s<16$ biquadrates, therefore, it is reasonable to restrict attention to integers $n$ with $n \equiv r(\bmod 16)$ for some integer $r$ with

2000 Mathematics Subject Classification: 11P05, 11P55.

Key words and phrases: Waring's problem, exceptional sets.

Research of T. D. Wooley supported in part by NSF grant DMS-9970440. 
$1 \leq r \leq s$. Motivated by such considerations, when $s \geq 5$ and $0 \leq t \leq s$, we define $E_{s, t}(X)$ to be the number of integers $n$ not exceeding $X$ that satisfy the congruence $n \equiv r(\bmod 16)$ for some integer $r$ with $1 \leq r \leq s-t$, yet cannot be written as the sum of $s$ biquadrates. Making use of a technical estimate, the proof of which is deferred to $\S 3$, we establish in $\S 2$ the estimates for $E_{s, 1}(X)$ recorded in the following theorem.

\section{Theorem 1.1. One has}

$$
E_{9,1}(X) \ll X^{27 / 55} \text { and } E_{10,1}(X) \ll X^{20 / 83} .
$$

In order to put the conclusion of Theorem 1.1 into an illuminating setting, we recall first that Vaughan [6] has shown that all large integers $n$, with $n \equiv r(\bmod 16)$ for some integer $r$ with $1 \leq r \leq 12$, may be written as the sum of 12 biquadrates, whence $E_{s, 0}(X) \ll 1$ for $s \geq 12$. Recent investigations of Kawada and Wooley [4], moreover, show that $E_{11,1}(X) \ll 1$ (see Theorem 3 of the latter paper). On applying Vaughan's work within conventional technology, one would obtain the bound

$$
E_{s, 0}(X) \ll X^{1-\delta-(s-6) / 16} \quad(s \geq 6),
$$

where $\delta$ is a number slightly larger than 0.0089 . The work of Kawada and Wooley, meanwhile, would lead to the estimate

$$
E_{s, 1}(X) \ll X^{1-(s-5) / 16+\varepsilon} \quad(s \geq 6),
$$

for each fixed $\varepsilon>0$. The superiority of Theorem 1.1 over the latter estimates is clear, although we emphasise that the bounds provided by (1.1) yield information concerning a congruence class not covered by the above theorem. At the cost of losing information concerning a second congruence class, a modest refinement of Theorem 1.1 may be achieved. Thus, making use of a technical estimate proved in $\S 5$, we establish in $\S 4$ the conclusion recorded in the following theorem.

\section{Theorem 1.2. For each positive number $\varepsilon$, one has}

$$
E_{9,2}(X) \ll X^{7 / 16+\varepsilon} \text { and } E_{10,2}(X) \ll X^{3 / 16+\varepsilon} .
$$

We advance in $\S \S 6$ and 7 to consider exceptional sets associated with sums of fifth powers, and here we are no longer plagued by local solubility considerations. When $s \geq 6$, define $\mathcal{E}_{s}(X)$ to be the number of natural numbers not exceeding $X$ that cannot be written as the sum of $s$ fifth powers of natural numbers. It follows from work of Vaughan and Wooley [9] that all large natural numbers are the sum of 17 positive fifth powers, whence $\mathcal{E}_{s}(X) \ll 1$ for $s \geq 17$. Indeed, the technology underlying the latter conclusion would permit the proof via classical methods of the upper bound

$$
\mathcal{E}_{s}(X) \ll X^{1-\sigma-(s-9) / 40} \quad(s \geq 9),
$$


for a certain number $\sigma$ slightly larger than 0.0095. Again making use of a technical estimate prepared in $\S 7$, we establish in $\S 6$ upper bounds for $\mathcal{E}_{s}(X)$ substantially sharper than those recorded in (1.2) for the cases $s=15$ and 16 .

\section{Theorem 1.3. One has}

$$
\mathcal{E}_{15}(X) \ll X^{62 / 105} \text { and } \mathcal{E}_{16}(X) \ll X^{17 / 30} .
$$

We remark that in the conclusions of both Theorems 1.1 and 1.3, a further modest reduction in the exponents should be feasible by exploiting efficient differencing methods restricted to minor arcs only, as will be familiar to aficionados of the modern Hardy-Littlewood method. Such improvements being in any case small, and requiring significant technical elaboration, it seems prudent to conserve space by instead encouraging energetic readers to explore such options elsewhere.

Throughout, the letter $\varepsilon$ will denote a sufficiently small positive number. We use $\ll$ and $\gg$ to denote Vinogradov's well-known notation, implicit constants depending at most on $\varepsilon$, unless otherwise indicated. In an effort to simplify our analysis, we adopt the convention that whenever $\varepsilon$ appears in a statement, then we are implicitly asserting that for each $\varepsilon>0$ the statement holds for sufficiently large values of the main parameter. Note that the "value" of $\varepsilon$ may consequently change from statement to statement, and hence also the dependence of implicit constants on $\varepsilon$.

2. The proof of Theorem 1.1: the nub. It is reasonably simple to describe the key elements of our proof of Theorem 1.1, and our aim in this section is to provide a complete account save for some technical discussion associated with the major arc treatment. Fundamental to our argument is the identity

$$
(x+y)^{4}+(x-y)^{4}+(2 y)^{4}=2\left(x^{2}+3 y^{2}\right)^{2},
$$

exploited first in the context of Waring's problem for biquadrates in earlier work of Kawada and Wooley [4].

Let $N$ be a large positive number, and let $s$ and $t$ be integers with $s \geq 5$ and $0 \leq t \leq s$. We denote by $\mathcal{N}_{s, t}$ the set of integers $n$ that satisfy the congruence $n \equiv r(\bmod 16)$ for some integer $r$ with $1 \leq r \leq s-t$. Define next $\mathcal{Z}_{s, t}(N)$ to be the set of integers $n \in \mathcal{N}_{s, t}$ with $N / 2<n \leq N$ that cannot be written as the sum of $s$ biquadrates. It is convenient to abbreviate $\operatorname{card}\left(\mathcal{Z}_{s, t}(N)\right)$ to $Z_{s, t}$. Next put $P=N^{1 / 4}$, and write $e(z)$ for $e^{2 \pi i z}$. We introduce the exponential sums

$$
f(\alpha)=\sum_{P / 3<z \leq P} e\left(\alpha z^{4}\right) \text { and } g(\alpha)=\sum_{\substack{P / 4 \leq x, y \leq P \\ x \neq y}} e\left(2\left(x^{2}+3 y^{2}\right)^{2} \alpha\right) .
$$


In order to make use of recent developments in the circle method involving the use of smooth numbers, we require some further notation. When $X$ and $Y$ are positive numbers, denote by $\mathcal{A}(X, Y)$ the set of $Y$-smooth numbers up to $X$, that is,

$$
\mathcal{A}(X, Y)=\{n \in[1, X] \cap \mathbb{Z}: p \mid n \text { and } p \text { prime implies that } p \leq Y\} .
$$

We then write

$$
h(\alpha ; X, Y)=\sum_{x \in \mathcal{A}(X, Y)} e\left(\alpha x^{4}\right) .
$$

We recall at this point some mean value estimates stemming from the sharpest available versions of the new iterative methods in Waring's problem.

LEMma 2.1. There is a positive number $\eta$ with the property that whenever $R \leq P^{\eta}$, one has

$$
\begin{aligned}
& \int_{0}^{1}\left|f(\alpha)^{2} h(\alpha ; P, R)^{4}\right| d \alpha \ll P^{3.18343}, \\
& \int_{0}^{1}\left|f(\alpha)^{2} h(\alpha ; P, R)^{8}\right| d \alpha \ll P^{6.21344} .
\end{aligned}
$$

Proof. On considering the underlying diophantine equations, the desired estimates are immediate from Theorem 2 of Brüdern and Wooley [2].

In the next section, and the remainder of this one, we suppose that $\eta$ is chosen sufficiently small that the conclusion of Lemma 2.1 holds. We then put $R=P^{\eta}$, and abbreviate $h(\alpha ; P, R)$ simply to $h(\alpha)$.

We now focus attention on the proof of Theorem 1.1. Suppose that $l$ is 0 or 1 , and write

$$
R_{1, l}(n)=\int_{0}^{1} g(\alpha) f(\alpha)^{2+l} h(\alpha)^{4} e(-n \alpha) d \alpha .
$$

Then on recalling the identity (2.1), it is apparent from (2.2)-(2.4) that whenever $s=9+l$ and $n \in \mathcal{Z}_{s, 1}(N)$, one has $R_{1, l}(n)=0$. Define the exponential sum

$$
K_{s, 1}(\alpha)=\sum_{n \in \mathcal{Z}_{s, 1}(N)} e(n \alpha)
$$

Then we conclude from (2.4) that

$$
\int_{0}^{1} g(\alpha) f(\alpha)^{2+l} h(\alpha)^{4} K_{s, 1}(-\alpha) d \alpha=\sum_{n \in \mathcal{Z}_{s, 1}(N)} R_{1, l}(n)=0 .
$$


We interpret the relation (2.5) by means of the Hardy-Littlewood method. Define the set of major arcs $\mathfrak{M}$ to be the union of the intervals

$$
\mathfrak{M}(q, a)=\left\{\alpha \in[0,1):|q \alpha-a| \leq P^{1 / 2} N^{-1}\right\},
$$

with $0 \leq a \leq q \leq P^{1 / 2}$ and $(a, q)=1$. We then denote the corresponding set of minor arcs by $\mathfrak{m}=[0,1) \backslash \mathfrak{M}$. In $\S 3$ below, we establish a lower bound for the contribution of the major arcs to the integral on the right hand side of (2.4), and this we summarise in the form of a lemma.

LEMMA 2.2. Suppose that $l$ is 0 or 1 , and that $n \in \mathcal{N}_{9+l, 1}$ satisfies $N / 2<$ $n \leq N$. Then

$$
\int_{\mathfrak{M}} g(\alpha) f(\alpha)^{2+l} h(\alpha)^{4} e(-n \alpha) d \alpha \gg N^{1+l / 4} .
$$

Assuming the validity of Lemma 2.2, we find that

$$
\int_{\mathfrak{M}} g(\alpha) f(\alpha)^{2+l} h(\alpha)^{4} K_{9+l, 1}(-\alpha) d \alpha \gg \sum_{n \in \mathcal{Z}_{9+l, 1}(N)} N^{1+l / 4}=Z_{9+l, 1} N^{1+l / 4}
$$

whence by (2.5),

$$
\left|\int_{\mathfrak{m}} g(\alpha) f(\alpha)^{2+l} h(\alpha)^{4} K_{9+l, 1}(-\alpha) d \alpha\right| \gg Z_{9+l, 1} N^{1+l / 4} .
$$

Our strategy is now to provide an upper bound for the left hand side of the inequality (2.7), and thereby obtain an upper bound for $Z_{9+l, 1}$. With this objective in mind, we record an auxiliary estimate of use also in our proof of Theorem 1.2. It is convenient, temporarily, to omit subscripts to the exponential sum $K_{s, t}(\alpha)$, the set $\mathcal{Z}_{s, t}(N)$, and the number $Z_{s, t}$.

LEMMA 2.3. For each positive number $\varepsilon$, one has

$$
\begin{gathered}
\int_{0}^{1}|g(\alpha) K(\alpha)|^{2} d \alpha \ll P^{\varepsilon}\left(Z^{2}+P^{2} Z\right), \\
\int_{0}^{1}|f(\alpha) g(\alpha) K(\alpha)|^{2} d \alpha \ll P^{\varepsilon}\left(P^{2} Z^{2}+P^{3} Z\right) .
\end{gathered}
$$

Proof. On recalling (2.2), we find from orthogonality that the integral on the left hand side of (2.8) is bounded above by the number of integral solutions of the system

$$
2\left(m_{1}^{2}-m_{2}^{2}\right)=n_{1}-n_{2}, \quad m_{i}=u_{i}^{2}+3 v_{i}^{2} \quad(i=1,2),
$$

with $1 \leq u_{i}, v_{i} \leq P(i=1,2)$ and $n_{l} \in \mathcal{Z}(N)(l=1,2)$. Given any one of the $O\left(Z^{2}\right)$ possible choices for $n_{1}$ and $n_{2}$ with $n_{1} \neq n_{2}$, an elementary divisor function estimate shows that there are $O\left(P^{\varepsilon}\right)$ possible choices for $m_{1}$ and $m_{2}$ satisfying $(2.10)$, whence $O\left(P^{2 \varepsilon}\right)$ possible choices for $u_{i}$ and $v_{i}$ $(i=1,2)$. When $n_{1}=n_{2}$, meanwhile, one has $u_{1}^{2}+3 v_{1}^{2}=u_{2}^{2}+3 v_{2}^{2}$, and 
an elementary divisor function estimate demonstrates on this occasion that there are $O\left(P^{2} \log P\right)$ possible choices for $u_{i}$ and $v_{i}(i=1,2)$. We therefore conclude that

$$
\int_{0}^{1}|g(\alpha) K(\alpha)|^{2} d \alpha \ll P^{2 \varepsilon} Z^{2}+Z P^{2} \log P,
$$

and the first desired conclusion (2.8) follows immediately.

Next observe that the integral on the left hand side of (2.9) is, by orthogonality, bounded above by the number of integral solutions of the system

$$
2\left(m_{1}^{2}-m_{2}^{2}\right)=n_{1}-n_{2}+x_{1}^{4}-x_{2}^{4}, \quad m_{i}=u_{i}^{2}+3 v_{i}^{2} \quad(i=1,2),
$$

with $1 \leq u_{i}, v_{i} \leq P(i=1,2), 1 \leq x_{j} \leq P(j=1,2)$ and $n_{l} \in \mathcal{Z}(N)$ $(l=1,2)$. Given any one of the $O\left(Z^{2} P^{2}\right)$ possible choices for $n_{1}, n_{2}, x_{1}, x_{2}$ with

$$
n_{1}+x_{1}^{4} \neq n_{2}+x_{2}^{4},
$$

an elementary divisor function estimate shows that there are $O\left(P^{\varepsilon}\right)$ possible choices for $m_{1}, m_{2}$ satisfying (2.11), whence $O\left(P^{2 \varepsilon}\right)$ possible choices for $u_{i}$ and $v_{i}(i=1,2)$. The number of solutions, $\mathcal{T}_{1}$, of this type therefore satisfies

$$
\mathcal{T}_{1} \ll P^{2+2 \varepsilon} Z^{2} .
$$

When

$$
n_{1}+x_{1}^{4}=n_{2}+x_{2}^{4},
$$

meanwhile, one has $u_{1}^{2}+3 v_{1}^{2}=u_{2}^{2}+3 v_{2}^{2}$ once again, and we deduce as before that there are $O\left(P^{2} \log P\right)$ possible choices for $u_{i}$ and $v_{i}(i=1,2)$. However, a moment of contemplation reveals that the number, $\mathcal{S}$, of integral solutions of (2.13), with $1 \leq x_{j} \leq P(j=1,2)$ and $n_{l} \in \mathcal{Z}(N)(l=1,2)$, satisfies

$$
\mathcal{S} \ll P^{\varepsilon} Z^{2}+P Z .
$$

The latter estimate is also an immediate consequence of Lemma 2.1 of Wooley [14] in the case $j=1$. Thus we deduce that the number of solutions, $\mathcal{T}_{2}$, of this second type satisfies

$$
\mathcal{T}_{2} \ll\left(P^{\varepsilon} Z^{2}+P Z\right) P^{2} \log P .
$$

On collecting together (2.12) and (2.14), we therefore deduce that

$$
\int_{0}^{1}|f(\alpha) g(\alpha) K(\alpha)|^{2} d \alpha \ll P^{2+2 \varepsilon} Z^{2}+P^{3+\varepsilon} Z,
$$

and this confirms the desired estimate (2.9).

We now return to the lower bound (2.7) and extract our upper bound for $Z_{9+l, 1}(l=0,1)$. Suppose first that $l=0$. We apply Schwarz's inequality 
to (2.7) and deduce that

$$
Z_{9,1} N \ll\left(\sup _{\alpha \in \mathfrak{m}}|f(\alpha)|\right) I_{1}^{1 / 2} I_{2}^{1 / 2}
$$

where

$$
I_{1}=\int_{0}^{1}\left|f(\alpha)^{2} h(\alpha)^{8}\right| d \alpha \quad \text { and } \quad I_{2}=\int_{0}^{1}\left|g(\alpha) K_{9,1}(\alpha)\right|^{2} d \alpha .
$$

Experts in the application of the circle method will recognise that, by combining Weyl's inequality with standard major arc estimates, one obtains the upper bound

$$
\sup _{\alpha \in \mathfrak{m}}|f(\alpha)| \ll P^{7 / 8+\varepsilon}
$$

(see Lemma 2.4 and Chapter 4 of Vaughan [7], or simply refer to Lemma 3 of Vaughan [5] for a detailed account). We therefore conclude from (2.15) and Lemmata 2.1 and 2.3 that

$$
Z_{9,1} N \ll P^{7 / 8+\varepsilon}\left(P^{6.21344}\right)^{1 / 2}\left(Z_{9,1}^{2}+P^{2} Z_{9,1}\right)^{1 / 2}
$$

whence

$$
Z_{9,1} \ll Z_{9,1} N^{-0.004}+Z_{9,1}^{1 / 2} N^{0.24544} .
$$

Consequently, one has $Z_{9,1} \ll N^{27 / 55}$, and the first estimate of Theorem 1.1 is obtained by summing over dyadic intervals.

Suppose next that $l=1$. On this occasion, an application of Schwarz's inequality to (2.7) yields

$$
Z_{10,1} N^{5 / 4} \ll\left(\sup _{\alpha \in \mathfrak{m}}|f(\alpha)|\right) I_{1}^{1 / 2} I_{3}^{1 / 2}
$$

where

$$
I_{3}=\int_{0}^{1}\left|f(\alpha) g(\alpha) K_{10,1}(\alpha)\right|^{2} d \alpha
$$

We therefore deduce from (2.16) and Lemmata 2.1 and 2.3 that

$$
Z_{10,1} N^{5 / 4} \ll P^{7 / 8+\varepsilon}\left(P^{6.21344}\right)^{1 / 2}\left(P^{2} Z_{10,1}^{2}+P^{3} Z_{10,1}\right)^{1 / 2} .
$$

Consequently, one has

$$
Z_{10,1} \ll Z_{10,1} N^{-0.004}+Z_{10,1}^{1 / 2} N^{0.12044},
$$

and we may conclude that $Z_{10,1} \ll N^{20 / 83}$. As in the first case, the second estimate of Theorem 1.1 now follows on summing over dyadic intervals.

3. The proof of Theorem 1.1: major arc treatment. For workers well-versed in modern incarnations of the Hardy-Littlewood method, our analysis of the major arc contribution in (2.4) will contain no surprises. We 
therefore launch our treatment immediately, pausing only to introduce some additional notation. When $a \in \mathbb{Z}, q \in \mathbb{N}$ and $\beta \in \mathbb{R}$, we write

$$
S(q, a)=\sum_{r=1}^{q} e\left(a r^{4} / q\right) \text { and } v(\beta)=\int_{P / 3}^{P} e\left(\beta \gamma^{4}\right) d \gamma .
$$

Also, we define the function $f^{*}(\alpha)$ for $\alpha \in[0,1)$ by putting

$$
f^{*}(\alpha)=q^{-1} S(q, a) v(\alpha-a / q)
$$

when $\alpha \in \mathfrak{M}(q, a) \subseteq \mathfrak{M}$, and by taking $f^{*}(\alpha)=0$ otherwise. We begin by replacing the exponential sum $f(\alpha)$, in the integral on the left hand side of (2.6), by its approximation $f^{*}(\alpha)$.

LEMMA 3.1. When $l$ is 0 or 1 , one has

$$
\int_{\mathfrak{M}} g(\alpha)\left(f(\alpha)^{2+l}-f^{*}(\alpha)^{2+l}\right) h(\alpha)^{4} e(-n \alpha) d \alpha \ll P^{27 / 7+l} .
$$

Proof. It follows from Theorem 4.1 of Vaughan [7] that whenever $\alpha \in$ $\mathfrak{M}(q, a) \subseteq \mathfrak{M}$, then

$$
\left|f(\alpha)-f^{*}(\alpha)\right| \ll P^{1 / 4+\varepsilon} .
$$

But for any natural number $r$, one has

$$
\left|f(\alpha)^{r}-f^{*}(\alpha)^{r}\right| \ll\left|f(\alpha)-f^{*}(\alpha)\right|\left(|f(\alpha)|^{r-1}+\left|f(\alpha)-f^{*}(\alpha)\right|^{r-1}\right),
$$

and so we deduce that

$$
\begin{aligned}
\int_{\mathfrak{M}} g(\alpha)\left(f(\alpha)^{2+l}-f^{*}(\alpha)^{2+l}\right) h(\alpha)^{4} e( & -n \alpha) d \alpha \\
& \ll P^{1 / 4+\varepsilon} I_{4}+P^{(2+l) / 4+\varepsilon} I_{5},
\end{aligned}
$$

where

$$
I_{4}=\int_{0}^{1}\left|g(\alpha) f(\alpha)^{1+l} h(\alpha)^{4}\right| d \alpha \quad \text { and } \quad I_{5}=\int_{0}^{1}\left|g(\alpha) h(\alpha)^{4}\right| d \alpha .
$$

On combining Schwarz's inequality with a trivial estimate for $f(\alpha)$, one finds that

$$
I_{4} \leq P^{l} I_{6}^{1 / 2} I_{7}^{1 / 2}
$$

where

$$
I_{6}=\int_{0}^{1}\left|g(\alpha)^{2} h(\alpha)^{4}\right| d \alpha \quad \text { and } \quad I_{7}=\int_{0}^{1}\left|f(\alpha)^{2} h(\alpha)^{4}\right| d \alpha .
$$

But on considering the underlying diophantine equation, it follows from Lemma 3.4 of Kawada and Wooley [4] that $I_{6} \ll P^{4+\varepsilon}$, and from Lemma 2.1 above one has $I_{7} \ll P^{3.19}$. Thus we conclude that

$$
I_{4} \ll P^{l}\left(P^{4+\varepsilon}\right)^{1 / 2}\left(P^{3.19}\right)^{1 / 2} \ll P^{18 / 5+l} .
$$


Meanwhile, again applying Schwarz's inequality, one finds that

$$
I_{5} \leq I_{6}^{1 / 2}\left(\int_{0}^{1}|h(\alpha)|^{4} d \alpha\right)^{1 / 2},
$$

so that by our previous estimate for $I_{6}$, together with an easy consequence of Hua's lemma, one obtains

$$
I_{5} \ll\left(P^{4+\varepsilon}\right)^{1 / 2}\left(P^{2+\varepsilon}\right)^{1 / 2} \ll P^{3+\varepsilon} .
$$

Finally, on collecting together (3.3)-(3.5), the conclusion of the lemma is immediate.

Our next step is to prune down to a narrow set of major arcs on which the behaviour of $h(\alpha)$ is well-understood, and this requires further notation and some estimates from the literature. When $X$ is a real number with $1 \leq X \leq P$, let $\mathfrak{N}(X)$ denote the union of the intervals

$$
\mathfrak{N}(q, a)=\left\{\alpha \in[0,1):|q \alpha-a| \leq X N^{-1}\right\},
$$

with $0 \leq a \leq q \leq X$ and $(a, q)=1$. For the sake of convenience, we write $\mathfrak{K}(X)=\mathfrak{N}(2 X) \backslash \mathfrak{N}(X)$. Finally, we write $L=(\log N)^{1 / 100}$, and take $\mathfrak{W}$ to be the union of the intervals

$$
\mathfrak{W}(q, a)=\left\{\alpha \in[0,1):|\alpha-a / q| \leq L N^{-1}\right\},
$$

with $0 \leq a \leq q \leq L$ and $(a, q)=1$. We then put

$$
R^{*}(n)=\int_{\mathfrak{W}} g(\alpha) f^{*}(\alpha)^{2+l} h(\alpha)^{4} e(-n \alpha) d \alpha
$$

LEMMA 3.2. When $l$ is 0 or 1 , one has

$$
\int_{\mathfrak{M}} g(\alpha) f(\alpha)^{2+l} h(\alpha)^{4} e(-n \alpha) d \alpha=R^{*}(n)+O\left(P^{4+l} L^{-1 / 3}\right) .
$$

Proof. Let $X$ be a positive number with $X \leq P^{1 / 2}$. It follows from partial integration that

$$
v(\beta) \ll P\left(1+P^{4}|\beta|\right)^{-1},
$$

and hence we may apply Lemma 5.4 of Vaughan and Wooley [10] to deduce that

$$
\int_{\mathfrak{N}(2 X)}\left|f^{*}(\alpha)^{2} h(\alpha)^{4}\right| d \alpha \ll X^{\varepsilon} P^{2} .
$$

Also, as a consequence of Lemma 3.3 of Kawada and Wooley [4], one has the upper bound

$$
\sup _{\alpha \in \mathfrak{K}(X)}|g(\alpha)| \ll P^{2} X^{\varepsilon-1 / 2},
$$


so that on making use of a trivial estimate for $f^{*}(\alpha)$, we find that

$$
\begin{aligned}
\int_{\mathfrak{K}(X)}\left|g(\alpha) f^{*}(\alpha)^{2+l} h(\alpha)^{4}\right| d \alpha & \ll P^{2+l} X^{\varepsilon-1 / 2} \int_{\mathfrak{N}(2 X)}\left|f^{*}(\alpha)^{2} h(\alpha)^{4}\right| d \alpha \\
& \ll P^{4+l} X^{-1 / 3} .
\end{aligned}
$$

Then on summing (3.6) over $X=2^{i} L$, with $i \geq 0$ and $X \leq P^{1 / 2}$, we deduce that

$$
\int_{\mathfrak{M} \backslash \mathfrak{W}} g(\alpha) f^{*}(\alpha)^{2+l} h(\alpha)^{4} e(-n \alpha) d \alpha \ll P^{4+l} L^{-1 / 3} .
$$

The proof is completed by recalling the conclusion of Lemma 3.1.

The proof of Lemma 2.2 is now easily completed with the use of familiar technology. Write

$$
w(\beta)=\int_{0}^{P} e\left(\beta \gamma^{4}\right) d \gamma,
$$

and define the function $h^{*}(\alpha)$ for $\alpha \in[0,1)$ by taking

$$
h^{*}(\alpha)=q^{-1} S(q, a) w(\alpha-a / q),
$$

when $\alpha \in \mathfrak{W}(q, a) \subseteq \mathfrak{W}$, and by taking $h^{*}(\alpha)=0$ otherwise. Then it follows from Lemma 8.5 of Wooley [11] (see also Lemma 5.4 of Vaughan [6] for a related conclusion) that there exists a positive number $c$, depending only on $\eta$, such that

$$
\sup _{\alpha \in \mathfrak{W}}\left|h(\alpha)-c h^{*}(\alpha)\right| \ll P L^{-10} .
$$

But the measure of $\mathfrak{W}$ is $O\left(L^{3} P^{-4}\right)$, and so it follows that

$$
\begin{aligned}
& R^{*}(n)-c^{4} \int_{\mathfrak{W}} g(\alpha) f^{*}(\alpha)^{2+l} h^{*}(\alpha)^{4} e(-n \alpha) d \alpha \\
& \ll\left(P^{8+l} L^{-10}\right)\left(L^{3} P^{-4}\right) \ll P^{4+l} L^{-7} .
\end{aligned}
$$

Write

$$
\begin{aligned}
I(m) & =\int_{-L P^{-4}}^{L P^{-4}} v(\beta)^{2+l} w(\beta)^{4} e(-m \beta) d \beta \\
\mathfrak{S}(m) & =\sum_{1 \leq q \leq L} \sum_{\substack{a=1 \\
(a, q)=1}}^{q}\left(q^{-1} S(q, a)\right)^{6+l} e(-m a / q) \\
\varrho(m) & =I(m) \mathfrak{S}(m) .
\end{aligned}
$$


Then it follows from the definitions of $g(\alpha), f^{*}(\alpha)$ and $h^{*}(\alpha)$ that

$$
\int_{\mathfrak{W}} g(\alpha) f^{*}(\alpha)^{2+l} h^{*}(\alpha)^{4} e(-n \alpha) d \alpha=\sum_{\substack{P / 4 \leq x, y \leq P \\ x \neq y}} \varrho\left(n-2\left(x^{2}+3 y^{2}\right)^{2}\right) .
$$

However, the partial singular series, $\mathfrak{S}(m)$, and singular integral, $I(m)$, are the same, in all essentials, as the corresponding quantities appearing in the familiar classical analysis of Waring's problem for sums of $6+l$ biquadrates. On applying the methods of Chapters 2 and 4 of Vaughan [7], therefore, one finds with little difficulty that there is a positive absolute constant $B$ with the property that

$$
\begin{cases}I(m) \geq-B P^{2+l} L^{-1 / 4} & \text { when } m \leq N / 4 \\ I(m) \gg m^{(2+l) / 4}+O\left(P^{2+l} L^{-1 / 4}\right) & \text { when } N / 4<m \leq N .\end{cases}
$$

Moreover, one has $\mathfrak{S}(m) \gg 1$ whenever $m \equiv r(\bmod 16)$ for some $r$ with $1 \leq r \leq 6+l$, and otherwise, for some positive absolute constant $C$, one has $\mathfrak{S}(m) \geq-C L^{-1 / 4}$. The same methods, meanwhile, ensure that in all circumstances one has $I(m) \ll P^{2+l}$ and $\mathfrak{S}(m) \ll 1$. Consequently, whenever $n \equiv r(\bmod 16)$ for some $r$ with $1 \leq r \leq 8+l$, and $N / 2<n \leq N$, it follows by considering values of $x$ and $y$ in appropriate residue classes modulo 2 that

$$
\sum_{\substack{P / 4 \leq x, y \leq P \\ x \neq y}} \varrho\left(n-2\left(x^{2}+3 y^{2}\right)^{2}\right) \gg P^{4+l}+O\left(P^{4+l} L^{-1 / 4}\right) .
$$

Finally, on collecting together the conclusion of Lemma 3.2 with (3.7)(3.9), we conclude that whenever $n \in \mathcal{N}_{9+l, 1}$ and $N / 2<n \leq N$, then

$$
\int_{\mathfrak{M}} g(\alpha) f(\alpha)^{2+l} h(\alpha)^{4} e(-n \alpha) d \alpha \gg P^{4+l}=N^{1+l / 4} .
$$

This establishes the lower bound (2.6), and hence completes the proof of Lemma 2.2.

4. More on sums of biquadrates. The proof of Theorem 1.2 may be accomplished with the tools developed in $\S \S 2$ and 3 through heavier use of the identity (2.1). We suppose that $l$ is a non-negative integer, and write

$$
R_{2, l}(n)=\int_{0}^{1} g(\alpha)^{2} f(\alpha)^{3+l} e(-n \alpha) d \alpha .
$$

Then on recalling the identity (2.1), it is apparent from (2.2) and (4.1) that whenever $s=9+l$ and $n \in \mathcal{Z}_{s, 2}(N)$, one has $R_{2, l}(n)=0$. Following the 
pattern established in $\S 2$, we define the exponential sum

$$
K_{s, 2}(\alpha)=\sum_{n \in \mathcal{Z}_{s, 2}(N)} e(n \alpha),
$$

and conclude from (4.1) that

$$
\int_{0}^{1} g(\alpha)^{2} f(\alpha)^{3+l} K_{s, 2}(-\alpha) d \alpha=0 .
$$

In $\S 5$ below we establish a lower bound for the contribution of the major arcs to the integral on the right hand side of (4.1), and this we record in the following lemma.

Lemma 4.1. Suppose that $l$ is 0 or 1 , and that $n \in \mathcal{N}_{9+l, 2}$ satisfies $N / 2<n \leq N$. Then

$$
\int_{\mathfrak{M}} g(\alpha)^{2} f^{*}(\alpha)^{3+l} e(-n \alpha) d \alpha \gg N^{(3+l) / 4} .
$$

Assuming the validity of Lemma 4.1, we deduce from (4.2) that

$$
\int_{\mathfrak{M}} g(\alpha)^{2} f^{*}(\alpha)^{3+l} K_{9+l, 2}(-\alpha) d \alpha \gg Z_{9+l, 2} N^{(3+l) / 4},
$$

whence by (4.3) we see that

$$
\left|T_{1}\right|+\left|T_{2}\right| \gg Z_{9+l, 2} N^{(3+l) / 4},
$$

where we write

$$
\begin{aligned}
& T_{1}=\int_{\mathfrak{m}} g(\alpha)^{2} f(\alpha)^{3+l} K_{9+l, 2}(-\alpha) d \alpha, \\
& T_{2}=\int_{\mathfrak{M}} g(\alpha)^{2}\left(f(\alpha)^{3+l}-f^{*}(\alpha)^{3+l}\right) K_{9+l, 2}(-\alpha) d \alpha .
\end{aligned}
$$

But in view of (3.1) and (3.2), one has

$$
T_{2} \ll P^{1 / 4+\varepsilon} I_{8, l}+P^{(3+l) / 4+\varepsilon} Z_{9+l, 2} I_{9},
$$

where

$$
I_{8, l}=\int_{0}^{1}\left|g(\alpha)^{2} f(\alpha)^{2+l} K_{9+l, 2}(\alpha)\right| d \alpha \quad \text { and } \quad I_{9}=\int_{0}^{1}|g(\alpha)|^{2} d \alpha .
$$

Furthermore, we find from (2.16) that

$$
T_{1} \ll\left(\sup _{\alpha \in \mathfrak{m}}|f(\alpha)|\right) \int_{0}^{1}\left|g(\alpha)^{2} f(\alpha)^{2+l} K_{9+l, 2}(\alpha)\right| d \alpha \ll P^{7 / 8+\varepsilon} I_{8, l} .
$$

Then we may conclude from (4.5)-(4.7) that

$$
P^{7 / 8+\varepsilon} I_{8, l}+P^{(3+l) / 4+\varepsilon} Z_{9+l, 2} I_{9} \gg Z_{9+l, 2} N^{(3+l) / 4} .
$$


Observe next that $I_{9}$ is bounded above by the number of integral solutions of the equation

$$
2\left(x_{1}^{2}+3 y_{1}^{2}\right)^{2}=2\left(x_{2}^{2}+3 y_{2}^{2}\right)^{2},
$$

with $1 \leq x_{i}, y_{i} \leq P(i=1,2)$. But for each fixed choice of $x_{2}$ and $y_{2}$, there are $O\left(P^{\varepsilon}\right)$ possible choices of $x_{1}$ and $y_{1}$ satisfying (4.9), and so we obtain the upper bound $I_{9} \ll P^{2+\varepsilon}$. On substituting the latter into (4.8), we deduce that

$$
Z_{9+l, 2} N^{(3+l) / 4} \ll P^{7 / 8+\varepsilon} I_{8, l}+P^{(11+l) / 4+\varepsilon} Z_{9+l, 2},
$$

whence

$$
Z_{9+l, 2} N^{(3+l) / 4} \ll P^{7 / 8+\varepsilon} I_{8, l} .
$$

Suppose now, in the first instance, that $l=0$. On applying Schwarz's inequality to the mean value $I_{8,0}$, we find that

$$
I_{8,0} \leq I_{10}^{1 / 2} I_{11}^{1 / 2}
$$

where

$$
I_{10}=\int_{0}^{1}\left|g(\alpha)^{2} f(\alpha)^{4}\right| d \alpha \quad \text { and } \quad I_{11}=\int_{0}^{1}\left|g(\alpha) K_{9,2}(\alpha)\right|^{2} d \alpha .
$$

But on considering the underlying diophantine equations, it follows from Lemma 3.4 of Kawada and Wooley [4] that $I_{10} \ll P^{4+\varepsilon}$. Moreover, Lemma 2.3 shows that $I_{11} \ll P^{\varepsilon}\left(Z_{9,2}^{2}+P^{2} Z_{9,2}\right)$. Thus we deduce from (4.10) and (4.11) that

$$
Z_{9,2} N^{3 / 4} \ll P^{7 / 8+\varepsilon}\left(P^{4+\varepsilon}\right)^{1 / 2}\left(Z_{9,2}^{2}+P^{2} Z_{9,2}\right)^{1 / 2},
$$

whence

$$
Z_{9,2} \ll Z_{9,2} P^{\varepsilon-1 / 8}+Z_{9,2}^{1 / 2} P^{7 / 8+\varepsilon} .
$$

We therefore obtain the upper bound $Z_{9,2} \ll N^{7 / 16+\varepsilon}$, and so the first estimate of Theorem 1.2 follows on summing over dyadic intervals.

Suppose next that $l=1$. Applying Schwarz's inequality once again, we now find from (4.10) that

$$
Z_{10,2} N \ll P^{7 / 8+\varepsilon} I_{10}^{1 / 2} I_{12}^{1 / 2}
$$

where

$$
I_{12}=\int_{0}^{1}\left|f(\alpha) g(\alpha) K_{10,2}(\alpha)\right|^{2} d \alpha .
$$

Then by the above estimate $I_{10} \ll P^{4+\varepsilon}$, and the second estimate of Lemma 2.3 , we obtain the upper bound

$$
Z_{10,2} N \ll P^{7 / 8+\varepsilon}\left(P^{4+\varepsilon}\right)^{1 / 2}\left(P^{2} Z_{10,2}^{2}+P^{3} Z_{10,2}\right)^{1 / 2} .
$$


Thus we conclude that

$$
Z_{10,2} \ll Z_{10,2} P^{\varepsilon-1 / 8}+Z_{10,2}^{1 / 2} P^{3 / 8+\varepsilon},
$$

and the estimate $Z_{10,2} \ll N^{3 / 16+\varepsilon}$ follows immediately. Finally, on summing over dyadic intervals, we obtain the second estimate of Theorem 1.2.

5. The proof of Theorem 1.2: major arc treatment. The pruning procedure associated with the proof of Lemma 4.1 is in many respects more straightforward than the corresponding treatment of $\S 3$, since there are no smooth Weyl sums to complicate matters. On the other hand, there are few conventional sums $f(\alpha)$ to assist us, and so the required conclusions cannot be lifted cheaply from the literature. We require some additional notation before replacing the sum $g(\alpha)$ by a suitable major arc approximation. Write

$$
\begin{aligned}
T(q, a) & =\sum_{r=1}^{q} \sum_{s=1}^{q} e\left(2 a\left(r^{2}+3 s^{2}\right)^{2} / q\right), \\
u(\beta) & =\int_{P / 4}^{P} \int_{P / 4}^{P} e\left(2\left(\xi^{2}+3 \zeta^{2}\right)^{2} \beta\right) d \xi d \zeta .
\end{aligned}
$$

Also, define the function $g^{*}(\alpha)$ for $\alpha \in[0,1)$ by putting

$$
g^{*}(\alpha)=q^{-2} T(q, a) u(\alpha-a / q)
$$

when $\alpha \in \mathfrak{M}(q, a) \subseteq \mathfrak{M}$, and by taking $g^{*}(\alpha)=0$ otherwise.

LEMMA 5.1. When $l$ is 0 or 1 , one has

$$
\int_{\mathfrak{M}}\left(g(\alpha)^{2}-g^{*}(\alpha)^{2}\right) f^{*}(\alpha)^{3+l} e(-n \alpha) d \alpha \ll P^{14 / 5+l} .
$$

Proof. Before launching our discussion of the upper bound (5.1), we require some preliminary estimates. First we note that, as a consequence of Lemma 3.3 of Kawada and Wooley [4], one has

$$
\sup _{\alpha \in \mathfrak{M}}\left|g(\alpha)-g^{*}(\alpha)\right| \ll P^{3 / 2+\varepsilon} \text {. }
$$

Next, from the same lemma, it follows that whenever $\alpha \in \mathfrak{M}(q, a) \subseteq \mathfrak{M}$,

$$
g^{*}(\alpha) \ll P^{2} q^{\varepsilon-1 / 2}\left(1+P^{4}|\alpha-a / q|\right)^{-1} .
$$

The latter upper bound, in particular, suffices to establish that

$$
\int_{\mathfrak{M}}\left|g^{*}(\alpha)\right|^{4} d \alpha \ll P^{4+\varepsilon} \text {. }
$$

Also, it follows from Lemma 3.5 of Kawada and Wooley [4] that

$$
\int_{0}^{1}|g(\alpha)|^{4} d \alpha \ll P^{4+\varepsilon} \text {. }
$$


Finally, from the argument of the proof of Lemma 5.1 of Vaughan [6], one obtains the upper bound

$$
\int_{\mathfrak{M}}\left|f^{*}(\alpha)\right|^{6} d \alpha \ll P^{2} .
$$

Returning now to the desired inequality (5.1), we note first that, in view of (5.2), one has

$$
\int_{\mathfrak{M}}\left(g(\alpha)^{2}-g^{*}(\alpha)^{2}\right) f^{*}(\alpha)^{3+l} e(-n \alpha) d \alpha \ll P^{3 / 2+\varepsilon}\left(I_{13}+I_{14}\right),
$$

where

$$
I_{13}=\int_{\mathfrak{M}}\left|g(\alpha) f^{*}(\alpha)^{3+l}\right| d \alpha \quad \text { and } \quad I_{14}=\int_{\mathfrak{M}}\left|g^{*}(\alpha) f^{*}(\alpha)^{3+l}\right| d \alpha .
$$

On applying Hölder's inequality within $I_{13}$, and making use of a trivial estimate for $f^{*}(\alpha)$, we find that

$$
I_{13} \ll P^{l}\left(\int_{0}^{1}|g(\alpha)|^{4} d \alpha\right)^{1 / 4}\left(\int_{\mathfrak{M}}\left|f^{*}(\alpha)\right|^{6} d \alpha\right)^{1 / 2}\left(\int_{\mathfrak{M}} d \alpha\right)^{1 / 4} .
$$

Then on noting that the measure of $\mathfrak{M}$ is $O\left(P^{-3}\right)$, and applying (5.5) and (5.6), we see that

$$
I_{13} \ll P^{l}\left(P^{4+\varepsilon}\right)^{1 / 4}\left(P^{2}\right)^{1 / 2}\left(P^{-3}\right)^{1 / 4} \ll P^{5 / 4+l+\varepsilon} .
$$

A comparison between (5.4) and (5.5) reveals that the same treatment, applied to $I_{14}$, yields the upper bound $I_{14} \ll P^{5 / 4+l+\varepsilon}$, and hence we conclude from (5.7) that

$$
\int_{\mathfrak{M}}\left(g(\alpha)^{2}-g^{*}(\alpha)^{2}\right) f^{*}(\alpha)^{3+l} e(-n \alpha) d \alpha \ll P^{11 / 4+l+\varepsilon},
$$

as desired. The conclusion of the lemma now follows immediately.

Write

$$
\begin{aligned}
I^{*}(q, n) & =\int_{-q^{-1} P^{-7 / 2}}^{q^{-1} P^{-7 / 2}} u(\beta)^{2} v(\beta)^{3+l} e(-n \beta) d \beta, \\
A^{*}(q, n) & =\sum_{\substack{a=1 \\
(a, q)=1}}^{q}\left(q^{-1} S(q, a)\right)^{3+l}\left(q^{-2} T(q, a)\right)^{2} e(-n a / q) .
\end{aligned}
$$

Then it follows from Lemma 5.1 that

$$
\int_{\mathfrak{M}} g(\alpha)^{2} f^{*}(\alpha)^{3+l} e(-n \alpha) d \alpha=\sum_{1 \leq q \leq P^{1 / 2}} I^{*}(q, n) A^{*}(q, n)+O\left(P^{14 / 5+l}\right) .
$$


Next define the complete singular integral $I^{*}(n)$ by

$$
I^{*}(n)=\int_{-\infty}^{\infty} u(\beta)^{2} v(\beta)^{3+l} e(-n \beta) d \beta .
$$

From Lemmata 3.1 and 3.3 of Kawada and Wooley [4], one has

$$
u(\beta)^{2} v(\beta)^{3+l} \ll P^{7+l}\left(1+P^{4}|\beta|\right)^{-2},
$$

and thus $I^{*}(n)$ is absolutely convergent with $I^{*}(n) \ll P^{3+l}$, and whenever $q \leq P^{1 / 2}$ one has the estimate

$$
I^{*}(q, n)-I^{*}(n) \ll P^{7+l} \int_{q^{-1} P^{-7 / 2}}^{\infty}\left(1+P^{4} \beta\right)^{-2} d \beta \ll P^{3+l}\left(q / P^{1 / 2}\right)^{1 / 5} .
$$

Furthermore, the standard theory of exponential sums shows that $A^{*}(q, n)$ is a multiplicative function of $q$ (see, for example, $\S 2.6$ of Vaughan [7]). Also, from Lemmata 3.2 and 3.3 of Kawada and Wooley [4], for each prime power $p^{h}$ one has

$$
A^{*}\left(p^{h}, n\right) \ll \begin{cases}p^{-3 / 2} & \text { when } h=1, \\ h^{2} p^{-3 h / 4} & \text { when } h>1 .\end{cases}
$$

Thus we find that

$$
\sum_{q=1}^{\infty}\left(q / P^{1 / 2}\right)^{1 / 5}\left|A^{*}(q, n)\right| \ll P^{-1 / 10} \text { and } \sum_{q=1}^{\infty}\left|A^{*}(q, n)\right| \ll 1 .
$$

On substituting (5.9) and (5.11) into (5.8), we conclude that

$$
\int_{\mathfrak{M}} g(\alpha)^{2} f^{*}(\alpha)^{3+l} e(-n \alpha) d \alpha=I^{*}(n) \mathfrak{S}^{*}(n)+O\left(P^{29 / 10+l}\right),
$$

where $\mathfrak{S}^{*}(n)$ denotes the singular series

$$
\mathfrak{S}^{*}(n)=\sum_{q=1}^{\infty} A^{*}(q, n)
$$

The proof of Lemma 4.1 is now almost complete. We remark next that a routine application of Fourier's integral theorem confirms that whenever $N / 2<n \leq N$, one has $I^{*}(n) \gg P^{3+l}$. Also, the upper bound for $A^{*}\left(p^{h}, n\right)$ provided by $(5.10)$ guarantees that $\mathfrak{S}^{*}(n)$ may be written as the absolutely convergent infinite product

$$
\mathfrak{S}^{*}(n)=\prod_{\varpi \text { prime }} \Omega_{\varpi}, \quad \text { where } \quad \Omega_{\varpi}=\sum_{h=0}^{\infty} A^{*}\left(\varpi^{h}, n\right) .
$$

Here, from (5.10), we have the estimate $\Omega_{\varpi}=1+O\left(\varpi^{-3 / 2}\right)$, and routine arguments show that whenever $n \in \mathcal{N}_{9+l, 2}$, one has $\Omega_{\varpi}>0$ (the reader may 
care to inspect pp. 189-190 of Kawada and Wooley [4] at this point). Thus we may conclude that $\mathfrak{S}^{*}(n) \gg 1$. Finally, on inserting the above estimates into (5.12), we arrive at the lower bound

$$
\int_{\mathfrak{M}} g(\alpha)^{2} f^{*}(\alpha)^{3+l} e(-n \alpha) d \alpha \gg P^{3+l}+O\left(P^{29 / 10+l}\right),
$$

valid whenever $N / 2<n \leq N$ and $n \in \mathcal{N}_{9+l, 2}$. This completes the proof of Lemma 4.1.

6. Sums of fifth powers. Following the pattern established above in our account of the proofs of Theorems 1.1 and 1.2, we defer to $\S 7$ a technical discussion of the major arc treatment associated with the proof of Theorem 1.3. The remaining part of the proof of Theorem 1.3 is straightforward to describe, and makes use of the identity

$$
\begin{aligned}
(h+x)^{5}+(h-x)^{5}+ & (h+y)^{5} \\
& +(h-y)^{5}+(h+x+y)^{5}+(h-x-y)^{5} \\
= & 20 h\left(x^{2}+x y+y^{2}+h^{2}\right)^{2}-14 h^{5}
\end{aligned}
$$

applied in earlier work of Kawada and Wooley [3] devoted to sums of fifth powers.

Let $N$ be a large positive number, and let $s$ be an integer with $s \geq 6$. We define $\mathcal{Z}_{s}(N)$ to be the set of integers $n$ with $N / 2<n \leq N$ that cannot be written as the sum of $s$ fifth powers. It is convenient in what follows to abbreviate $\operatorname{card}\left(\mathcal{Z}_{s}(N)\right)$ to $Z_{s}$. At the slight risk of confusion, we now discard our earlier notation, and write $P=N^{1 / 5}$. We then introduce the exponential sums

$$
\begin{aligned}
f(\alpha) & =\sum_{P / 2<u \leq P} e\left(\alpha u^{5}\right), \\
G(\alpha) & =\sum_{P / 2<z \leq P} \sum_{1 \leq x, y \leq P / 6} e(\Phi(x, y, z) \alpha),
\end{aligned}
$$

where we write

$$
\Phi(x, y, z)=20 z\left(x^{2}+x y+y^{2}+z^{2}\right)^{2}-14 z^{5} .
$$

Also, with the definition of $\mathcal{A}(X, Y)$ from $\S 2$, we now take

$$
h(\alpha ; X, Y)=\sum_{x \in \mathcal{A}(X, Y)} e\left(\alpha x^{5}\right)
$$

For future reference, we recall the mean value estimates recorded in the following lemma.

Lemma 6.1. There is a positive number $\eta$ with the property that whenever $R \leq P^{\eta}$, one has 


$$
\begin{array}{r}
\int_{0}^{1}|h(\alpha ; P, R)|^{6} d \alpha \ll P^{3.136258}, \\
\int_{0}^{1}\left|f(\alpha)^{2} h(\alpha ; P, R)^{6}\right| d \alpha \ll P^{4.438657}, \\
\int_{0}^{1}\left|f(\alpha)^{2} h(\alpha ; P, R)^{14}\right| d \alpha \ll P^{11.077363} .
\end{array}
$$

Proof. On considering the underlying diophantine equations, the desired estimates are immediate from the table on p. 236 of Vaughan and Wooley [9].

In this section and the next, we suppose that $\eta$ is chosen sufficiently small that the upper bounds recorded in Lemma 6.1 hold. We then put $R=P^{\eta}$, and abbreviate $h(\alpha ; P, R)$ to $h(\alpha)$.

We may now discuss the proof of Theorem 1.3 in earnest. Suppose that $l$ is 0 or 1 , and write

$$
R_{l}(n)=\int_{0}^{1} G(\alpha) f(\alpha)^{2+l} h(\alpha)^{7} e(-n \alpha) d \alpha .
$$

In view of the identity (6.1), it follows from (6.2)-(6.4) that whenever $s=$ $15+l$ and $n \in \mathcal{Z}_{s}(N)$, then one has $R_{l}(n)=0$. Define the exponential sum

$$
K_{s}(\alpha)=\sum_{n \in \mathcal{Z}_{s}(N)} e(n \alpha)
$$

Then we deduce from (6.4) that

$$
\int_{0}^{1} G(\alpha) f(\alpha)^{2+l} h(\alpha)^{7} K_{s}(-\alpha) d \alpha=0 .
$$

We next apply the Hardy-Littlewood method. Define the set of major $\operatorname{arcs} \mathfrak{M}$ now to be the union of the intervals

$$
\mathfrak{M}(q, a)=\left\{\alpha \in[0,1):|q \alpha-a| \leq P^{5 / 16} N^{-1}\right\}
$$

with $0 \leq a \leq q \leq P^{5 / 16}$ and $(a, q)=1$. We then denote the corresponding set of minor arcs by $\mathfrak{m}=[0,1) \backslash \mathfrak{M}$. In $\S 7$ below, we provide a lower bound for the contribution of the major arcs on the right hand side of (6.4), and this we record in the form of a lemma.

LEMMA 6.2. Suppose that $l$ is 0 or 1 , and that $n$ is an integer with $N / 2<n \leq N$. Then

$$
\int_{\mathfrak{M}} G(\alpha) f(\alpha)^{2+l} h(\alpha)^{7} e(-n \alpha) d \alpha \gg N^{(7+l) / 5} .
$$


Assuming the validity of Lemma 6.2, we find that

$$
\int_{\mathfrak{M}} G(\alpha) f(\alpha)^{2+l} h(\alpha)^{7} K_{15+l}(-\alpha) d \alpha \gg Z_{15+l} N^{(7+l) / 5},
$$

whence by $(6.5)$,

$$
\left|\int_{\mathfrak{m}} G(\alpha) f(\alpha)^{2+l} h(\alpha)^{7} K_{15+l}(-\alpha) d \alpha\right| \gg Z_{15+l} N^{(7+l) / 5} .
$$

In order to bound $Z_{15+l}$, we require a mean value estimate analogous to the first of those established in Lemma 2.3 above. As before, we temporarily omit subscripts to the exponential sum $K_{s}(\alpha)$, the set $\mathcal{Z}_{s}(N)$, and the number $Z_{s}$.

Lemma 6.3. For each positive number $\varepsilon$, one has

$$
\int_{0}^{1}|G(\alpha) K(\alpha)|^{2} d \alpha \ll P^{\varepsilon}\left(P Z^{2}+P^{4} Z\right) .
$$

Proof. By Cauchy's inequality, one has

$$
\int_{0}^{1}|G(\alpha) K(\alpha)|^{2} d \alpha \leq P \sum_{P / 2<z \leq P 0} \int_{0}^{1}\left|H_{z}(\alpha) K(\alpha)\right|^{2} d \alpha,
$$

where we write

$$
H_{z}(\alpha)=\sum_{1 \leq x, y \leq P / 6} e(\Phi(x, y, z) \alpha) .
$$

Then by orthogonality, we find that the integral on the left hand side of (6.8) is bounded above by $P I_{15}$, where $I_{15}$ denotes the number of integral solutions of the system

$$
20 z\left(m_{1}^{2}-m_{2}^{2}\right)=n_{1}-n_{2}, \quad m_{i}-z^{2}=x_{i}^{2}+x_{i} y_{i}+y_{i}^{2} \quad(i=1,2),
$$

with $1 \leq z \leq P, 1 \leq x_{i}, y_{i} \leq P(i=1,2)$ and $n_{l} \in \mathcal{Z}(N)(l=1,2)$. Given any one of the $O\left(Z^{2}\right)$ possible choices for $n_{1}$ and $n_{2}$ with $n_{1} \neq n_{2}$, an elementary divisor function estimate shows that there are $O\left(P^{\varepsilon}\right)$ possible choices for $z, m_{1}$ and $m_{2}$ satisfying (6.9), whence $O\left(P^{2 \varepsilon}\right)$ possible choices for $z$ and $x_{i}, y_{i}(i=1,2)$. When $n_{1}=n_{2}$, on the other hand, one has

$$
x_{1}^{2}+x_{1} y_{1}+y_{1}^{2}=x_{2}^{2}+x_{2} y_{2}+y_{2}^{2},
$$

and an elementary divisor function estimate demonstrates that there are $O\left(P^{2} \log P\right)$ possible choices for $x_{i}, y_{i}(i=1,2)$. On accounting for the additional $O(P)$ possible choices for $z$ in the latter case, we arrive at the estimate

$$
I_{15} \ll P^{2 \varepsilon} Z^{2}+Z P^{3} \log P,
$$


whence

$$
\int_{0}^{1}|G(\alpha) K(\alpha)|^{2} d \alpha \ll P^{1+\varepsilon}\left(Z^{2}+Z P^{3}\right) .
$$

This establishes the conclusion of the lemma.

We are now equipped to complete our proof of Theorem 1.3. Suppose first that $l=0$. On applying Schwarz's inequality to (6.7), we find that

$$
Z_{15} N^{7 / 5} \ll\left(\sup _{\alpha \in \mathfrak{m}}|f(\alpha)|\right) I_{16}^{1 / 2} I_{17}^{1 / 2}
$$

where

$$
I_{16}=\int_{0}^{1}\left|f(\alpha)^{2} h(\alpha)^{14}\right| d \alpha \quad \text { and } \quad I_{17}=\int_{0}^{1}\left|G(\alpha) K_{15}(\alpha)\right|^{2} d \alpha .
$$

By combining Weyl's inequality with standard major arc estimates (see Lemma 2.4 and Chapter 4 of Vaughan [7]), one obtains

$$
\sup _{\alpha \in \mathfrak{m}}|f(\alpha)| \ll P^{15 / 16+\varepsilon} .
$$

Thus we deduce from (6.10) and Lemmata 6.1 and 6.3 that

$$
Z_{15} N^{7 / 5} \ll P^{15 / 16+\varepsilon}\left(P^{11.077363}\right)^{1 / 2}\left(P Z_{15}^{2}+P^{4} Z_{15}\right)^{1 / 2},
$$

whence

$$
Z_{15} \ll Z_{15} N^{-0.004}+Z_{15}^{1 / 2} N^{0.295237} .
$$

We therefore conclude that $Z_{15} \ll N^{62 / 105}$, and thus the first estimate of Theorem 1.3 follows on summing over dyadic intervals.

Next consider the situation in which $l=1$. An application of Schwarz's inequality to (6.7) now yields the upper bound

$$
Z_{16} N^{8 / 5} \ll\left(\sup _{\alpha \in \mathfrak{m}}|f(\alpha)|\right)^{2} I_{16}^{1 / 2} I_{17}^{1 / 2} .
$$

Consequently, it follows from (6.11) and Lemmata 6.1 and 6.3 that

$$
Z_{16} N^{8 / 5} \ll\left(P^{15 / 16+\varepsilon}\right)^{2}\left(P^{11.077363}\right)^{1 / 2}\left(P Z_{16}^{2}+P^{4} Z_{16}\right)^{1 / 2},
$$

from which we obtain

$$
Z_{16} \ll Z_{16} N^{-0.017}+Z_{16}^{1 / 2} N^{0.282737}
$$

Thus we conclude that $Z_{16} \ll N^{17 / 30}$, and the second estimate of Theorem 1.3 follows by summing over dyadic intervals.

7. The proof of Theorem 1.3: major arc treatment. The major arc treatment required for the proof of Theorem 1.3 is complicated by the presence of the unusual exponential sum $G(\alpha)$. Rather than deal directly with the latter sum, we instead employ the remaining $9+l$ more conventional exponential sums within (6.6) to obtain a useable major arc analysis, and 
then sum over the variables implicit in $G(\alpha)$. The treatment therefore shares many features in common with that described in $\S 3$. We begin our discussion with some additional notation. When $a \in \mathbb{Z}, q \in \mathbb{N}$ and $\beta \in \mathbb{R}$, we write

$$
S(q, a)=\sum_{r=1}^{q} e\left(a r^{5} / q\right) \text { and } v(\beta)=\int_{P / 2}^{P} e\left(\beta \gamma^{5}\right) d \gamma
$$

Also, we define the function $f^{*}(\alpha)$ for $\alpha \in[0,1)$ by putting

$$
f^{*}(\alpha)=q^{-1} S(q, a) v(\alpha-a / q)
$$

when $\alpha \in \mathfrak{M}(q, a) \subseteq \mathfrak{M}$, and by taking $f^{*}(\alpha)=0$ otherwise.

Lemma 7.1. When $l$ is 0 or 1 , one has

$$
\int_{\mathfrak{M}} G(\alpha)\left(f(\alpha)^{2+l}-f^{*}(\alpha)^{2+l}\right) h(\alpha)^{7} e(-n \alpha) d \alpha \ll P^{69 / 10+l} .
$$

Proof. From Theorem 4.1 of Vaughan [7], we find that whenever $\alpha \in$ $\mathfrak{M}(q, a) \subseteq \mathfrak{M}$, one has

$$
\left|f(\alpha)-f^{*}(\alpha)\right| \ll P^{5 / 32+\varepsilon} .
$$

On recalling the general inequality (3.2), we deduce that

$$
\begin{aligned}
\int_{\mathfrak{M}} G(\alpha)\left(f(\alpha)^{2+l}-f^{*}(\alpha)^{2+l}\right) & h(\alpha)^{7} e(-n \alpha) d \alpha \\
& \ll P^{5 / 32+\varepsilon} I_{18}+P^{5(2+l) / 32+\varepsilon} I_{19},
\end{aligned}
$$

where

$$
I_{18}=\int_{0}^{1}\left|G(\alpha) f(\alpha)^{1+l} h(\alpha)^{7}\right| d \alpha \quad \text { and } \quad I_{19}=\int_{0}^{1}\left|G(\alpha) h(\alpha)^{7}\right| d \alpha .
$$

On making use of a trivial estimate for $f(\alpha)$, it follows from Schwarz's inequality that

$$
I_{18} \leq P^{l} I_{20}^{1 / 2} I_{21}^{1 / 2}
$$

where

$$
I_{20}=\int_{0}^{1}\left|G(\alpha)^{2} h(\alpha)^{8}\right| d \alpha \quad \text { and } \quad I_{21}=\int_{0}^{1}\left|f(\alpha)^{2} h(\alpha)^{6}\right| d \alpha .
$$

But on considering the underlying diophantine equation, it follows from Lemma 3.1 of Kawada and Wooley [3] that $I_{20} \ll P^{9+\varepsilon}$, and from Lemma 6.1 one has $I_{21} \ll P^{4.44}$. Thus we conclude that

$$
I_{18} \ll P^{l}\left(P^{9+\varepsilon}\right)^{1 / 2}\left(P^{4.44}\right)^{1 / 2} \ll P^{6.73+l} .
$$


Meanwhile, again applying Schwarz's inequality, one obtains

$$
I_{19} \leq I_{20}^{1 / 2}\left(\int_{0}^{1}|h(\alpha)|^{6} d \alpha\right)^{1 / 2},
$$

so that our previous bound for $I_{20}$, combined with the first estimate of Lemma 6.1, yields

$$
I_{19} \ll\left(P^{9+\varepsilon}\right)^{1 / 2}\left(P^{3.14}\right)^{1 / 2} \ll P^{6.08} .
$$

The conclusion of the lemma follows on combining the bounds (7.1)-(7.3).

Recall next the definitions of the $\operatorname{arcs} \mathfrak{N}(X), \mathfrak{K}(X)$ and $\mathfrak{W}$ from $\S 3$. We seek to prune back the set of arcs $\mathfrak{M}$ to the narrow set of arcs $\mathfrak{W}$ on which we have good control of the smooth Weyl sums $h(\alpha)$. We now put

$$
R^{*}(n)=\int_{\mathfrak{W}} G(\alpha) f^{*}(\alpha)^{2+l} h(\alpha)^{7} e(-n \alpha) d \alpha .
$$

LEMma 7.2. When $l$ is 0 or 1 , one has

$$
\int_{\mathfrak{M}} G(\alpha) f(\alpha)^{2+l} h(\alpha)^{7} e(-n \alpha) d \alpha=R^{*}(n)+O\left(P^{7+l} L^{-1 / 4}\right) .
$$

Proof. By partial integration one has

$$
v(\beta) \ll P\left(1+P^{5}|\beta|\right)^{-1},
$$

and so we may apply Lemma 5.4 of Vaughan and Wooley [10] to deduce that whenever $1 \leq X \leq P^{5 / 16}$, one has

$$
\int_{\mathfrak{N}(2 X)}\left|f^{*}(\alpha)^{2} h(\alpha)^{4}\right| d \alpha \ll X^{\varepsilon} P .
$$

Also, on combining the conclusions of Lemmata 7.2 and 8.5 of Vaughan and Wooley [8], one finds that

$$
\sup _{\alpha \in \mathfrak{K}(X)}|h(\alpha)| \ll P X^{-1 / 11} .
$$

Consequently, on making use of trivial estimates for $f^{*}(\alpha)$ and $G(\alpha)$, it follows that

$$
\begin{array}{rl}
\int_{\mathfrak{K}(X)} \mid G(\alpha) f^{*}(\alpha)^{2+l} & h(\alpha)^{7} \mid d \alpha \\
& \ll P^{3+l}\left(\sup _{\alpha \in \mathfrak{K}(X)}|h(\alpha)|\right)^{3} \int_{\mathfrak{N}(2 X)}\left|f^{*}(\alpha)^{2} h(\alpha)^{4}\right| d \alpha \\
& \ll P^{7+l} X^{-1 / 4} .
\end{array}
$$

The proof of the lemma is completed on summing (7.4) over $X=2^{i} L$, with $i \geq 0$ and $X \leq P^{5 / 16}$, and recalling the conclusion of Lemma 7.1. 
The proof of Lemma 6.2 now lies within our grasp. Write

$$
w(\beta)=\int_{0}^{P} e\left(\beta \gamma^{5}\right) d \gamma,
$$

and define the function $h^{*}(\alpha)$ for $\alpha \in[0,1)$ by taking

$$
h^{*}(\alpha)=q^{-1} S(q, a) w(\alpha-a / q),
$$

when $\alpha \in \mathfrak{W}(q, a) \subseteq \mathfrak{W}$, and by taking $h^{*}(\alpha)=0$ otherwise. Then it follows from Lemma 8.5 of Wooley [11] that there exists a positive number $c$, depending only on $\eta$, such that

$$
\sup _{\alpha \in \mathfrak{W}}\left|h(\alpha)-c h^{*}(\alpha)\right| \ll P L^{-10} .
$$

Since the measure of $\mathfrak{W}$ is $O\left(L^{3} P^{-5}\right)$, we find that

$$
\begin{aligned}
R^{*}(n)-c^{7} \int_{\mathfrak{W}} G(\alpha) f^{*}(\alpha)^{2+l} h^{*}(\alpha)^{7} e(-n \alpha) d \alpha \\
\\
\ll\left(P^{12+l} L^{-10}\right)\left(L^{3} P^{-5}\right) \ll P^{7+l} L^{-7} .
\end{aligned}
$$

Write

$$
\begin{aligned}
I(m) & =\int_{-L P^{-5}}^{L P^{-5}} v(\beta)^{2+l} w(\beta)^{7} e(-m \beta) d \beta, \\
\mathfrak{S}(m) & =\sum_{1 \leq q \leq L} \sum_{\substack{a=1 \\
(a, q)=1}}^{q}\left(q^{-1} S(q, a)\right)^{9+l} e(-m a / q), \\
\varrho(m) & =I(m) \mathfrak{S}(m) .
\end{aligned}
$$

Then it follows from the definitions of $G(\alpha), f^{*}(\alpha)$ and $h^{*}(\alpha)$ that

$$
\begin{aligned}
\int_{\mathfrak{W}} G(\alpha) f^{*}(\alpha)^{2+l} h^{*}(\alpha)^{7} e & (-n \alpha) d \alpha \\
& =\sum_{P / 2<z \leq P} \sum_{1 \leq x, y \leq P / 6} \varrho(n-\Phi(x, y, z)) .
\end{aligned}
$$

But the partial singular series $\mathfrak{S}(m)$, and singular integral $I(m)$, are essentially the same as the corresponding quantities appearing in the familiar classical analysis of Waring's problem for sums of $9+l$ fifth powers. On applying the methods of Chapters 2 and 4 of Vaughan [7], we therefore deduce that there is a positive absolute constant $B$ with the property that

$$
\begin{cases}I(m) \geq-B P^{4+l} L^{-1 / 5} & \text { when } m \leq N / 4 \\ I(m) \gg m^{(4+l) / 5}+O\left(P^{4+l} L^{-1 / 5}\right) & \text { when } N / 4<m \leq N .\end{cases}
$$

Also, one deduces that $\mathfrak{S}(m) \gg 1$ uniformly in $m$. The same methods, on the other hand, demonstrate that in all cases one has $I(m) \ll P^{4+l}$ and 
$\mathfrak{S}(m) \ll 1$. Consequently, whenever $N / 2<n \leq N$, it follows that

$$
\sum_{P / 2<z \leq P} \sum_{1 \leq x, y \leq P / 6} \varrho(n-\Phi(x, y, z)) \gg P^{7+l}+O\left(P^{7+l} L^{-1 / 5}\right) .
$$

Finally, on collecting together the conclusion of Lemma 7.2 with (7.5)(7.7), we conclude that whenever $N / 2<n \leq N$, one has

$$
\int_{\mathfrak{M}} G(\alpha) f(\alpha)^{2+l} h(\alpha)^{7} e(-n \alpha) d \alpha \gg P^{7+l} \gg n^{(7+l) / 5},
$$

and this leads immediately to the desired lower bound (6.6), thereby completing the proof of Lemma 6.2.

\section{References}

[1] J. Brüdern, K. Kawada and T. D. Wooley, Additive representation in thin sequences, I: Waring's problem for cubes, Ann. Sci. École Norm. Sup. (4) 34 (2001), 471-501.

[2] J. Brüdern and T. D. Wooley, On Waring's problem: two cubes and seven biquadrates, Tsukuba J. Math. 24 (2000), 387-417.

[3] K. Kawada and T. D. Wooley, Sums of fifth powers and related topics, Acta Arith. 87 (1998), 27-65.

[4] - - - Sums of fourth powers and related topics, J. Reine Angew. Math. 512 (1999), $173-223$.

[5] R. C. Vaughan, On Waring's problem for smaller exponents. II, Mathematika 33 (1986), 6-22.

[6] -, A new iterative method in Waring's problem, Acta Math. 162 (1989), 1-71.

[7] -, The Hardy-Littlewood Method, 2nd ed., Cambridge Univ. Press, Cambridge, 1997.

[8] R. C. Vaughan and T. D. Wooley, On Waring's problem: some refinements, Proc. London Math. Soc. (3) 63 (1991), 35-68.

[9] —, - Further improvements in Waring's problem, Acta Math. 174 (1995), 147-240.

[10] - - - Further improvements in Waring's problem, IV: Higher powers, Acta Arith. 94 (2000), 203-285.

[11] T. D. Wooley, On simultaneous additive equations, II, J. Reine Angew. Math. 419 (1991), 141-198.

[12] - Slim exceptional sets for sums of four squares, Proc. London Math. Soc. (3), in press.

[13] -, Slim exceptional sets for sums of cubes, Canad. J. Math., in press.

[14] - Slim exceptional sets and the asymptotic formula in Waring's problem, Math. Proc. Cambridge Philos. Soc., to appear.

Department of Mathematics

Faculty of Education

Iwate University

Morioka 020-8550, Japan

E-mail: kawada@iwate-u.ac.jp
Department of Mathematics University of Michigan East Hall, 525 East University Avenue Ann Arbor, MI 48109-1109, U.S.A. E-mail: wooley@math.lsa.umich.edu 